\title{
Contacto temprano del alumno de medicina con su realidad de salud. Experiencia peruana
}

\author{
Alejandro Bermúdez-García, Claudia Mory-Arciniega
}

Recientemente, Baños et al [1] publicaron en $E d u$ cación Médica la experiencia piloto del nuevo grado conjunto de medicina de la Universitat Autònoma de Barcelona y la Universitat Pompeu Fabra en relación con la exposición precoz a la realidad sociosanitaria de los estudiantes y su satisfacción. Nuestra experiencia en la Escuela de Medicina de la Facultad de Ciencias de la Salud de la Universidad Peruana de Ciencias Aplicadas, en Lima, ratifica lo planteado por los autores.

Desde hace cuatro años, como parte del plan de estudios, los alumnos del primer semestre visitan los diferentes centros asistenciales correspondientes al campo clínico de nuestra escuela. Estas visitas están dirigidas principalmente a que los alumnos logren cuatro objetivos: conocer el sistema de salud peruano, sensibilizar al alumno con la realidad del paciente como persona que sufre, iniciar el desarrollo de la competencia de comunicación y conocer los determinantes de la salud en nuestro medio.

Los estudiantes visitan desde puestos de salud en zonas periféricas de la ciudad con equipamiento mínimo hasta institutos especializados con alta tecnología, así como instituciones públicas y privadas. Este contraste les permite entender las diferentes realidades en las cuales podrán desempeñar su labor. Creemos que este momento, en el que el alumno comienza a descubrir el sistema de salud nacional y a la vez construye la visión de la comunidad, es muy útil para desarrollar un pensamiento crítico sobre la realidad a la que se enfrentará y, según esta, empezar a alinear su concepción sobre el cuidado de la salud.

El alumno inicia las entrevistas con pacientes intentando entender todo lo que les ocurre a su alrededor en el momento de sufrir una enfermedad; para ello utilizamos el modelo clásico de los determinantes de salud desarrollado por Lalonde-Laframboise [2]. De esa forma, pueden ver no solo la enfermedad, sino considerar el paciente como producto de un determinante biológico (en lo referido a herencia, maduración, envejecimiento y desarrollo de los sistemas), los determinantes del medio ambiente (físicos, químicos, biológicos y psicosociales), los hábitos de vida como causantes de enfermedad (decisiones asumidas y hábitos nocivos) y la organización de los servicios de salud en su población (acceso a la prevención, curación y rehabilitación). Estas actividades han permitido a los alumnos reforzar su vocación e incrementar su grado de compromiso y responsabilidad.

Debemos destacar entonces que los alumnos de medicina han de tener un contacto precoz con los sistemas de salud de su comunidad para poder sentar las bases necesarias de su formación.

Bibliografía

1. Baños JE, Sentí M, Miralles R. Contacto precoz con la realidad asistencial: una experiencia piloto en medicina. Educ Med 2011; 14: 39-47.

2. Lalonde M. A new perspective of the health of Canadians. A working document. Ottawa: Minister of Supply and Services; 1981.
Escuela de Medicina. Universidad Peruana de Ciencias Aplicadas. Lima, Perú.

Correspondencia:

Dr. Alejandro Bermúdez García. Escuela de Medicina. Universidad Peruana de Ciencias Aplicadas. Avda. Primavera, 2390. Lima, Perú.

E-mail:

alejandro.bermudez@upc.edu.pe

(C) 2011 Educación Médica 